\title{
Reduction of Electromagnetic Interference Using ZnO-PCL Nanocomposites at Microwave Frequency
}

\author{
Abubakar Yakubu, ${ }^{1}$ Zulkifly Abbas, ${ }^{1}$ Nor Azowa Ibrahim, ${ }^{2}$ and Ahmad Fahad ${ }^{3}$ \\ ${ }^{1}$ Department of Physics, Universiti Putra Malaysia (UPM), 43400 Serdang, Selangor, Malaysia \\ ${ }^{2}$ Department of Chemistry, Universiti Putra Malaysia (UPM), 43400 Serdang, Selangor, Malaysia \\ ${ }^{3}$ Institute of Mathematical Research (INSPEM), Universiti Putra Malaysia (UPM), 43400 Serdang, Malaysia
}

Correspondence should be addressed to Abubakar Yakubu; abulect73@yahoo.com

Received 9 March 2015; Revised 21 May 2015; Accepted 26 May 2015

Academic Editor: Markku Leskela

Copyright (C 2015 Abubakar Yakubu et al. This is an open access article distributed under the Creative Commons Attribution License, which permits unrestricted use, distribution, and reproduction in any medium, provided the original work is properly cited.

In industrial equipment and home appliance applications, the electromagnetic compatibility compliance directive (ECCD) demands that electromagnetic interference side effects be eliminated or marginally minimized. The equipment must not disturb radio and telecommunication as well as other appliances. Additionally the ECCD also governs the immunity of such equipment to interference and seeks to ensure that this equipment is not disturbed by radio emissions when used as intended. Many types of absorbing materials are commercially available. However, many are expensive and not environmentally friendly. It is in the light of the above that we studied the electromagnetic absorption properties of ZnO-PCL nanocomposites prepared from cheap and abundant resources which are environmentally friendly (zinc and polycaprolactone). The test was carried out using a microstrip, open ended coaxial probe, and vector network analyzer. Amongst other findings, result showed that the ZnO-PCL nanocomposite has the capability of attenuating microwave frequency up to $-18.2 \mathrm{~dB}$ due to their very high specific surface areas attributed to the nanofillers at $12 \mathrm{GHz}$.

\section{Introduction}

The organic-inorganic hybrid materials possess interesting functions through the amalgamation of the important properties from both components [1-3]. Nanocomposites based on oxide-polymer mixtures have been an active area of recent research, owing to their range of applications in microwave absorption and electromagnetic interference shielding (EMI) [4].

Nanocomposites are a special class of materials having unique properties and wide application potential in diverse areas [5]. The term nanocomposites can be obtained by successfully joining together two different materials in a single matrix. The addition of material in the nanometer scale will in no small measure change the properties of the host polymer matrices. It is reported that, to enhance the apparent dielectric constant of polymer nanodielectrics, a significant amount ( $>30 \mathrm{vol} \%)$ of spherical nanoparticles needs to be incorporated into the polymer matrix [6].
Oxide-polymer nanocomposites possess unique physical and chemical properties compared to their bulk counter parts due to their nanodimension. In comparison to traditional composites, nanocomposites are certainly advantageous in homogeneous structure, no fibre rupture, optical transparency, and improved or unchanged processability [7].

Some of the main factors which determine the absorption properties of the composites are the quality of the dispersion, dielectric properties, and the filler matrix interaction. Processing methods suitable for nanocomposites as well as their applications have been developed by in situ reactive blending, melt-mixing, thermokinetic mixing, extrusion, blowing, and injection $[7,8]$. Materials such as polyethylene, polypropylene and polyethylene terephthalate, epoxy, polyamide, and polyimide have been used in nanocomposites as polymers. Some of the fillers used in pioneering researches involving polymer oxides nanocomposites include $\mathrm{Al}_{2} \mathrm{O}_{3}, \mathrm{TiO}_{2}$, and $\mathrm{SiO}_{2}$. The major drawback faced with the earlier researches was the cost of raw materials used. $\mathrm{ZnO}$ as one of the 
multifunctional inorganic particles has drawn increasing attention in recent years due to its many significant physical and chemical stabilities, high catalytic activity, effective antibacterial and bactericide function, and intensive ultraviolet and infrared absorption [9, 10]. For these reasons, it is selected to serve as filler in its nanoform in the polymer composites. On the basis of the above description, this paper will investigate the use of $\mathrm{ZnO}$ nanoparticles as filler in the oxide-polymer composites, processing methods, experimental work, and results and discussion on electromagnetic properties as it affects radiation attenuation.

\section{Processing Method}

For the preparation of the nanocomposites used in this study, the following were used: zinc oxide $(\mathrm{ZnO}) 99.9 \%$ purity, with density of $5.606 \mathrm{~g} / \mathrm{cm}^{3}$ (Sigma, Aldrich), ethylenediaminetetraacetic acid (EDTA) $99.8 \%$ purity, with density of $860 \mathrm{mg} / \mathrm{mL}$ (Sigma Aldrich), polycaprolactone (PCL) $\left(\mathrm{C}_{6} \mathrm{H}_{10} \mathrm{O}_{2}\right) 97.0 \%$ purity, with density of $1.146 \mathrm{~g} / \mathrm{cm}^{3}$ (Sigma Aldrich), deionized water (DIW) $\left(\mathrm{H}_{2} \mathrm{O}\right)$ with density $1.0 \mathrm{~g} / \mathrm{cm}^{3}$, and polyvinyl alcohol (PVA), 86.89\% purity (Alfa Aesar). In our work, the $\mathrm{ZnO}-\mathrm{PCL}$ nanocomposites were prepared via the melt blend technique using the Thermo Haake extruder polydrive three-phase motor with a drive of $1.5 \mathrm{~kW}, 3 \times 230 \mathrm{~V}$, and $40 \mathrm{~A}$ and speed range of $0-120 \mathrm{rpm}$. The melt blend method is characterized by the fact that organicinorganic materials are made at relatively low temperatures $\left(80^{\circ} \mathrm{C}\right)$ and, in principle, consists of mixing of constituent materials and subsequent polycondensation to jelly-like form before hardening after cooling. It allows incorporation of organic and inorganic additives which is rather physical than chemical at low temperature. Mix blending is started from melting of the polymer in a Thermo Haake blending machine at $80^{\circ} \mathrm{C}$. After melting in the machine for about 10 minutes, required amount of nanofiller is introduced into the chamber where the polymer is kept. The process is allowed to rotate in the machine for 20 minutes, before it is taken out and fabricated to required dimension using the hot or cold moulding method. The process is repeated for different percentages of the nanofiller and polymer. The melt blend method is suitable for mass production. However, it is not the key technology for composites preparation. The biggest advantage of the melt blend technique is that it is very easy to use. The picture of prepared $\mathrm{ZnO}$-PCL nanocomposites pellets after undergoing the melt blend process is shown in Figure 1.

The absorption properties and chemical bonding of the composites were verified using Fourier transform infrared spectrophotometer (FTIR) Perkin 69 Elmer in the range of 380 to $4000 \mathrm{~cm}^{-1}$. The $\mathrm{ZnO}$ nanoparticles prepared were analyzed using the Hitachi 7100 TEM (Tokyo, Japan) at $75 \mathrm{kV}$. For microwave properties measurement, Agilent 85070B dielectric probe kit, a sensor probe, a mounting bracket, a cable, a 3.5 inch high density shorting block for calibration, adapters, and a software for data collection and plotting were used. The network vector analyzer model used was Agilent PNA-L N5230A. The calibration for the complex permittivity measurement was performed using the through-reflect-line calibration module.

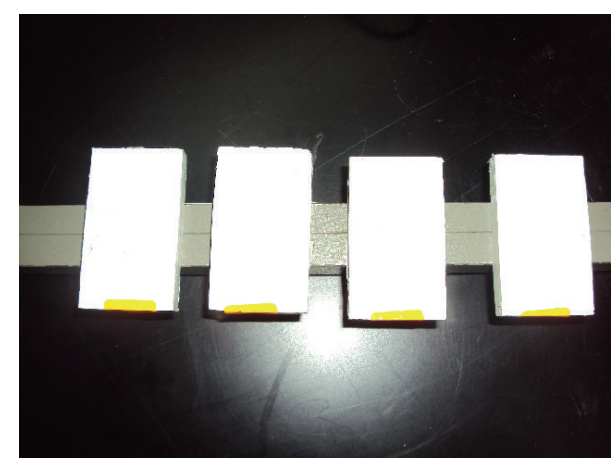

Figure 1: Prepared pellets of ZnO-PCL nanocomposites.

\section{Measurement Technique}

The material properties were studied in X-band $(8-12 \mathrm{GHz})$ regions of the microwave frequency spectra. The dimension of the microstrip used is $6.0 \times 3.6 \mathrm{~cm}$ with thickness of $8 \mathrm{~mm}$. The sample dimensions were fabricated carefully to match the microstrip dimensions. A vector network analyzer based microstrip measurement technique was employed to measure $S$-parameters of the two-port network formed by placing the sample on top of the microstrip whilst the open ended coaxial probe was used to measure the dielectric properties of the samples under study. $S$-parameters measured by the vector network analyzer were used to calculate the absorption and attenuation of the sample. The accuracy of the constitutive material properties depends on the accuracy with which $S$-parameters are measured. The through-reflect-line (TRL) calibration method was used to eliminate the systematic errors occurring in the measurement, since the experimental setup involves several components such as cables and connectors. Thus, proper care has to be taken to ensure that the entire system remains stable over the measurement period reducing reflection losses to be less than $\mathrm{dB}$. This technique has been successfully applied to measure common thin film materials and ferrite powders [11, 12]. The measurement theory, calibration, and data evaluation method used in our experimental setup does not require initial guess parameters, which reduces the error arising due to phase ambiguity.

\section{Result on Electromagnetic Properties}

The sample was measured by transmission-reflection waveguide technique in $\mathrm{X}$-band from 8 to $12 \mathrm{GHz}$ after using through-reflect-line calibration. The spectra for measured value of scattering parameters are shown in Figure 2. These values were used to calculate the attenuation of the $\mathrm{ZnO}$ PCL nanocomposites. It is expected that at higher frequency there is high attenuation of electromagnetic waves which propagates through sample, thereby leading to diminished transmitted waves. More so, based on the impedance mismatch theory, the higher the dielectric properties, the lower $\left|S_{21}\right|$ magnitude [13]. In addition, if the materials have higher loss factor then they tend to absorb more energy of the electromagnetic waves that were propagating through the 


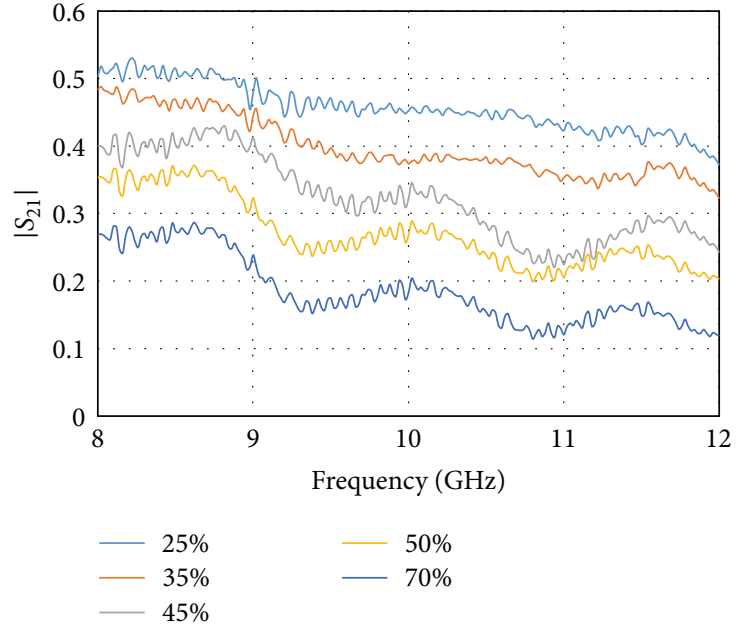

(a)

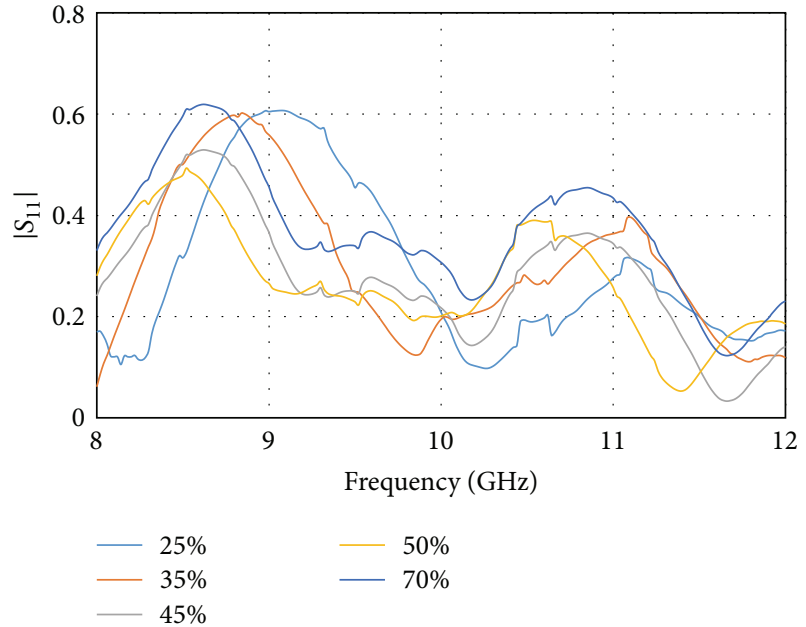

(b)

Figure 2: Variation in S-parameter using microstrip at X-band.

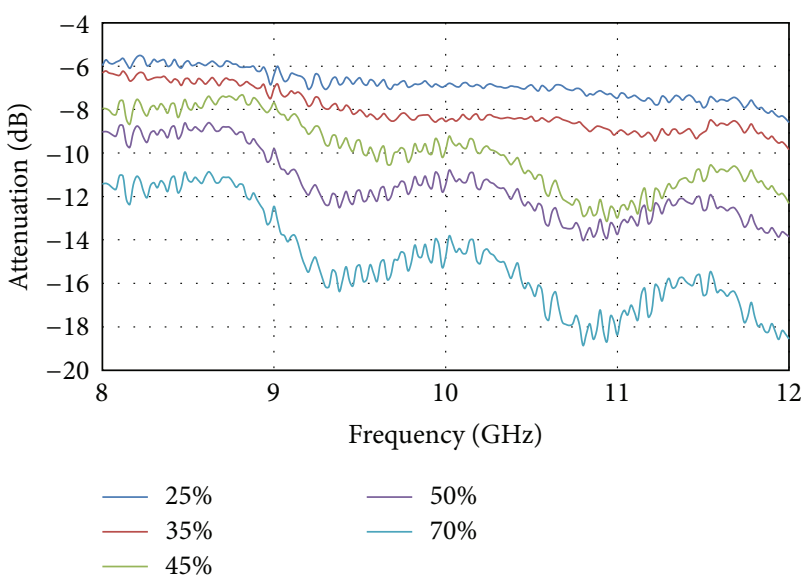

FIgURE 3: Attenuation for different $\mathrm{ZnO}$-PCL nanocomposites.

sample resulting in a diminished $S_{21}$ magnitude [13]. Figure 2 depicts the theory as reported by Pozar [13].

$S_{21}$ magnitude behaviour for the composites can be further confirmed by the analysis undertaken using the FTIR spectral analysis (Figure 8) which showed that the highest absorption (attenuation) was for the highest percentage $\mathrm{ZnO}$ PCL nanocomposites (70\% $\mathrm{ZnO}$ nanofiller). Higher concentration of filler is reported to result in formation of filler multilayer inside the polymer that reduces the intensity of light passing through polymer films as is evident in Figure 2. Besides multilayer effect, it was also suggested that the interparticle distance of nanofiller decreases as the amount of nanofiller in polymer nanocomposites increases [14]. The $\mathrm{X}$-band spectra for attenuation were calculated using the formula [13]

$$
\text { Attenuation }=20 \log \left(S_{21}\right) \text {. }
$$

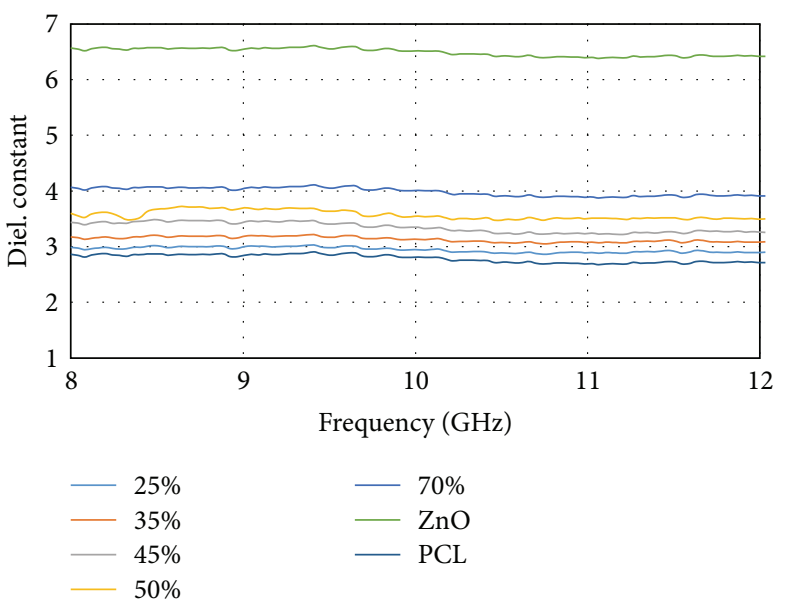

FIGURE 4: Variation in dielectric constant of samples.

The calculated attenuation of the $\mathrm{ZnO}$-PCL nanocomposites is shown in Figure 3.

An attenuator is a radio frequency $(\mathrm{RF})$ device specifically designed to reduce the power of a signal without affecting or reducing the waveform of the signal and for impedance matching due to its absorptive and dissipative nature. In electronic devices, standard attenuation values can be ranged from -0 to $-60 \mathrm{~dB}$ maximum attenuation with frequency maximums that range from $1 \mathrm{GHz}$ to $50 \mathrm{GHz}$ depending on attenuators type [15]. Observation on Figure 3 showed that attenuation from the different composites ranges from -6 to $-18.2 \mathrm{~dB}$. There was a clear indication of a sharp increase in attenuation as filler content increased to above $50 \%$. This increase is associated with the reduced particle sizes which results in an increase in the zinc oxide specific surface area, thus providing good interaction between the crosslinking agent particles and the polymer chains, thereby increasing attenuation as the $\mathrm{ZnO}$ nanofiller increases [16]. 


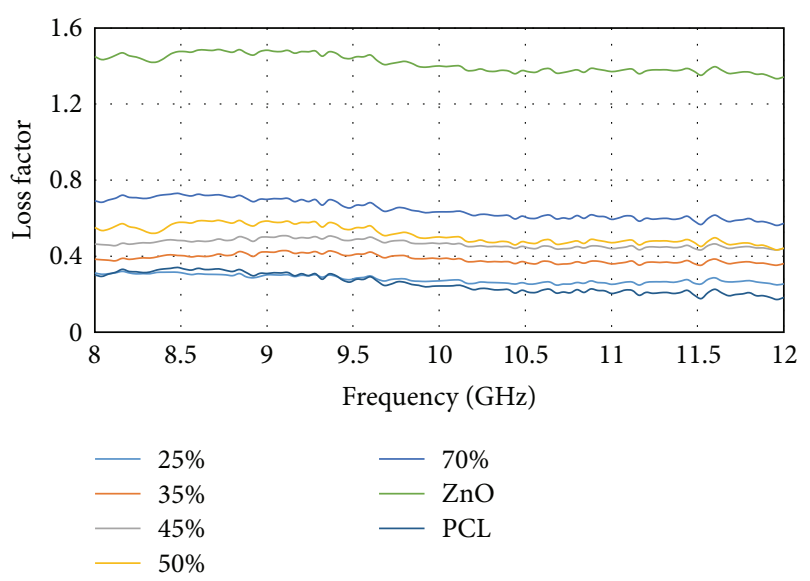

Figure 5: Variation in loss factor of samples.

TABLE 1: Real and imaginary parts of permittivity for all samples at $8-12 \mathrm{GHz}$.

\begin{tabular}{lccc}
\hline Sample & Dielectric const. & Loss factor & $\varepsilon_{r}=\varepsilon^{\prime}-j \varepsilon^{\prime \prime}$ \\
\hline Pure PCL & 2.79 & 0.24 & $2.79-j 0.24$ \\
ZnO nano & 6.48 & 1.41 & $6.48-j 1.41$ \\
ZnO-PCL-25\%ZnO & 2.95 & 0.27 & $2.95-j 0.27$ \\
ZnO-PCL-35\%ZnO & 3.15 & 0.39 & $3.15-j 0.39$ \\
ZnO-PCL-45\%ZnO & 3.34 & 0.46 & $3.34-j 0.46$ \\
ZnO-PCL-50\%ZnO & 3.54 & 0.50 & $3.54-j 0.50$ \\
ZnO-PCL-70\%ZnO & 3.99 & 0.63 & $3.99-j 0.63$ \\
\hline
\end{tabular}

The measured attenuation values fall within the range of low power attenuators in microwave devices. However, the composite percentage can be tailored to provide attenuation above $-20 \mathrm{~dB}$ which is mostly used in microwave devices. Higher attenuation of the composite can be achieved by increasing the percentage of the filler in the composites leading to increased level of RF attenuation and low magnitude of RF leakage. It is important to note that attenuation can vary greatly depending on the frequency of operation. In conclusion, depending on the application, the composites can be tailored to suit the application for which it is intended.

It is reported that materials with higher permittivity values tend to possess higher attenuation at higher frequency ranges. To understand the phenomena, the dielectric properties of the composites were measured. The real permittivity of the $\mathrm{ZnO}$ nanoparticles, pure PCL, and $\mathrm{ZnO}-\mathrm{PCL}$ nanocomposite showing decrease in dielectric constant as frequency increases is presented in Figure 4. This decrease in magnitude may be attributed to the real permittivity dispersion which is an interfacial polarization. This interfacial polarization can occur since the two materials have different permittivity and conductivity; hence when electrical field is applied to the composite, space charges provided by the $\mathrm{Zn}^{2+}$ phase accumulate at the interface of the two materials. This behavior is not usually intrinsic but is rather associated with the heterogeneous conduction in the multiphase structure of the composite [17].
The increase in dielectric magnitude of the $\mathrm{ZnO}-\mathrm{PCL}$ nanocomposites is also due to the number of $\mathrm{Zn}^{2+}$ added to the polymer matrix. The increase in the number of charged ions within the composite has contributed to the increase in the real permittivity of the $\mathrm{ZnO}$-PCL nanocomposites, whilst the loss factor shown in Figure 5 depicts the loss in energy of the propagated waves through the samples. The result showed that the $70 \% \mathrm{ZnO}-\mathrm{PCL}$ nanocomposites had the lowest loss which is in good agreement with the result obtained for the scattering parameters and FTIR analysis. In addition, the dielectric measurement result confirms the integration of the particle into the polymer matrix.

The higher percentage of nanofiller used in this work was done so as to produce composites whose dielectric constant would be close to the value of $\mathrm{ZnO}$ nanoparticle. The objective of higher filler content in the polymer matrix is to boost the complex permittivity value of the nanocomposites, which in turn leads to higher attenuation as reported by Pozar in [13]. The use of higher percentage of $\mathrm{ZnO}$ nanofiller in the composites is also supported by the work carried by [6] The effect of using higher value of filler is clearly expressed in Table 1. Careful observation on Table 1 showed that $70 \% \mathrm{ZnO}$ nanofiller has successfully enhanced the dielectric constant of the composites which is about $61.2 \%$ the value of the dielectric constant of the $\mathrm{ZnO}$ nanoparticles.

For the magnetic permeability shown in Figure 6, it was observed that the difference from one weight percent to other was very small. The value of permeability from the lowest to highest $\mathrm{ZnO}$ nanofiller is 1.034 to 1.041 . However, the permeability values are higher than unity because $8 \mathrm{GHz}$ lies in the low frequency tail of the broad magnetic resonance absorption band appearing at lower microwave frequencies. The higher the percentage of the $\mathrm{ZnO}$ nanofiller, the higher the values of the permeability, $\mu^{\prime}$. The infinitesimal change in the real part of permeability for all the composites might be attributed to the homogeneous nature of the composites attributed to good sample preparation technique [18].

However, the imaginary part of permeability shown in Figure 7 exhibits a significant change in magnitude for the different (\%) ZnO-PCL nanocomposites as frequency increases. The result showed a general increase of about $2 / 5$ times from $10 \mathrm{GHz}$ to $12 \mathrm{GHz}$ which is attributed to the increase in the $\mathrm{ZnO}$ nanofiller content.

All the analyses undertaken were further supported using a tool called the FTIR. The FTIR is used to confirm the bonding, interaction, and most of all the absorbing properties of the composites. The principal peaks from FTIR analysis for the $\mathrm{ZnO}-\mathrm{PCL}$ nanocomposite after nanoinclusion shown in Figure 8 are the $280 \mathrm{~cm}^{-1}$ peak due to the $\mathrm{Zn}-\mathrm{O}-\mathrm{Zn}$ stretching vibrations, the $730 \mathrm{~cm}^{-1}$ peak due to long chain rocking vibrations, the $1200 \mathrm{~cm}^{-1}$ peak due to C-O-C aliphatic ether stretching vibrations, the $1726 \mathrm{~cm}^{-1}$ peak due to the C-O stretching vibrations, the $2941 \mathrm{~cm}^{-1}$ peak due to $\mathrm{CH}_{2}$ stretching vibrations, and additional new peak at $3470 \mathrm{~cm}^{-1}$. The new isolated band peak exhibited by the ZnO-PCL nanocomposites at $3470 \mathrm{~cm}^{-1}$ may be attributed to $\mathrm{O}-\mathrm{H}$ species in the composites $[19,20]$. The lattice vibration bands between 3470 and $3420 \mathrm{~cm}^{-1}, 3070$ and $2720 \mathrm{~cm}^{-1}$, and 455 and $755 \mathrm{~cm}^{-1}$ of 


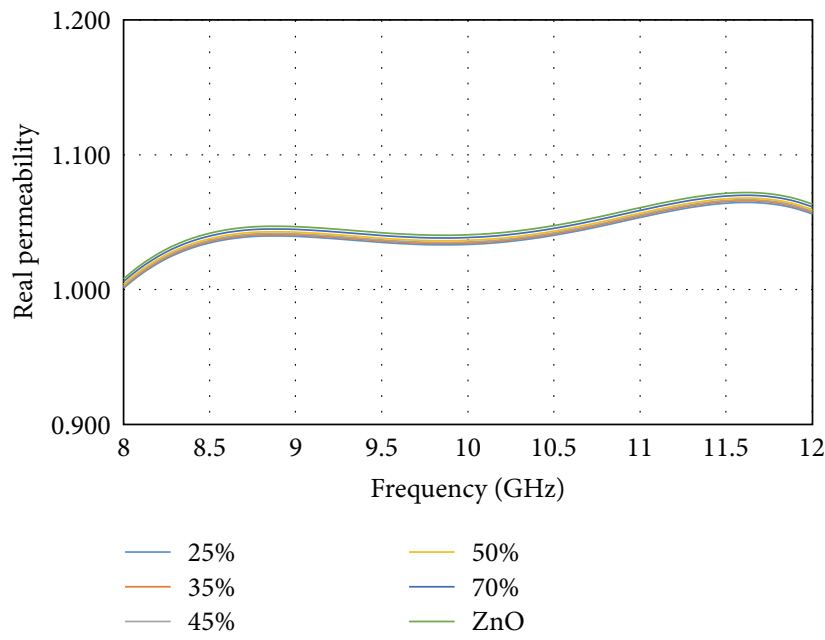

(a)

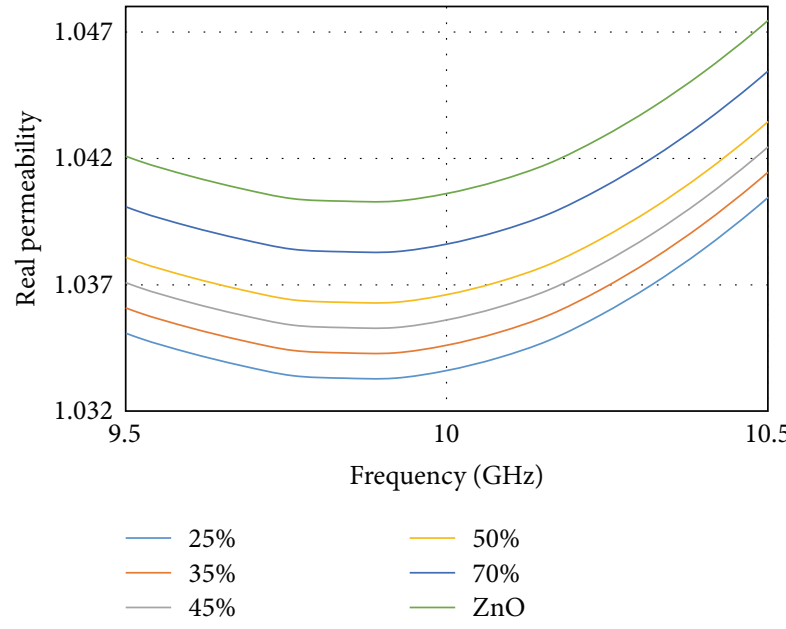

(b)

FIGURE 6: Variation in real permeability of ZnO-PCL nanocomposites.

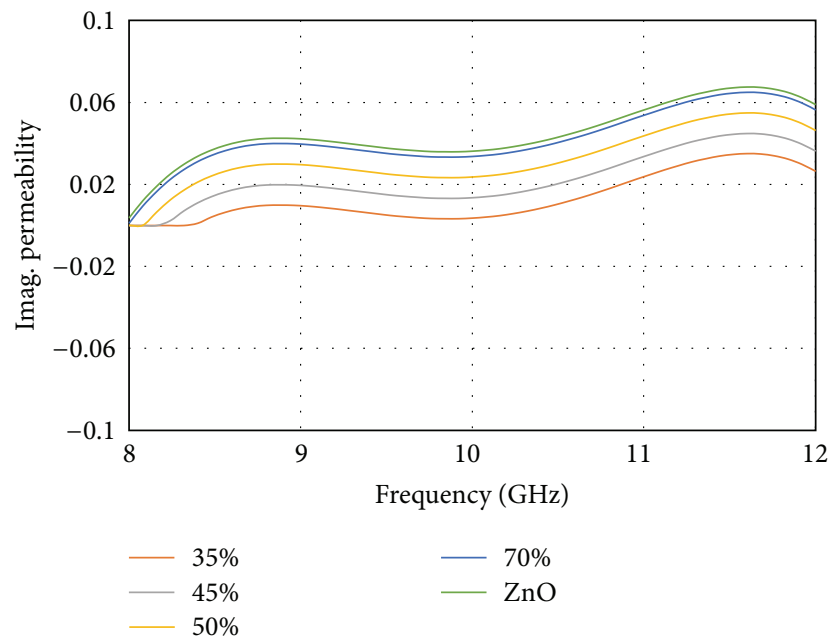

FIGURE 7: Variation in imaginary permeability of ZnO-PCL nanocomposites.
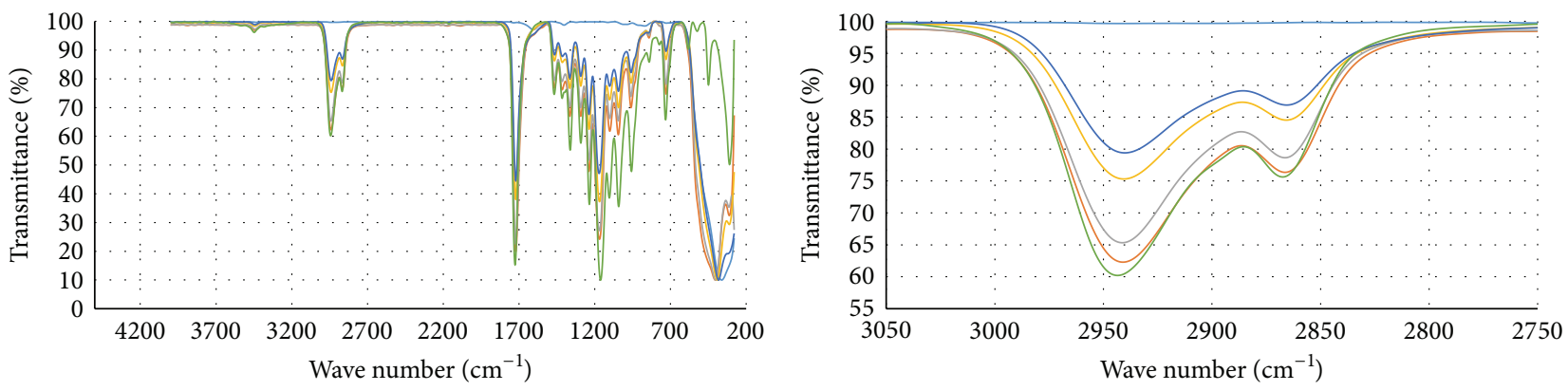

$\mathrm{ZnO}$
$-\quad 25 \%$
$-35 \%$

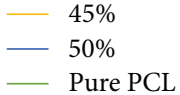

(a)

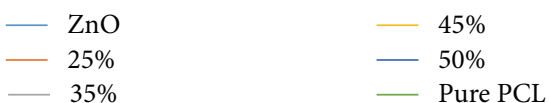

(b)

FIGURE 8: FTIR spectrum for different (\%) of $\mathrm{ZnO}$-PCL nanocomposites, $\mathrm{ZnO}$ and PCL. 
the pure PCL when compared to the $\mathrm{ZnO}$ nanoparticles fillers showed an increase in absorption peaks for all weight percent, thus providing evidence that the $\mathrm{ZnO}$ layers have been doped into the PCL matrix by the melt blending process, thereby forming the $\mathrm{ZnO}$-PCL nanocomposites as clearly shown in Figure 8(b). The increase in $\mathrm{ZnO}$ nanofiller increases the absorption peaks of the composites as is evident in [21].

TEM analysis was carried out so as to gain knowledge on the particle size distribution of the prepared $\mathrm{ZnO}$ particles. The analysis showed spherical particle size distribution of $\mathrm{ZnO}$ nanoparticles that was dispersed in the polymer. The average particles size was calculated to be $41.5 \mathrm{~nm}$.

\section{Error Analysis}

Experimental uncertainties (random errors) were minimized by repeating each measurement six times for each sample. Some other components identified that can cause random errors in measurement are the connectors between the network analyzer and microstrip, which can make the system sensitive to noise and drift. These errors can be minimized by keeping the whole system clean and stable, whilst systematic errors caused by the imperfections of the system were reduced by careful calibration of the whole measurement system before the actual measurements were undertaken.

\section{Conclusion}

The microstrip based transmission-reflection measurement technique has been successfully applied to calculate the attenuation of ZnO-PCL nanocomposites in the wide frequency range of $8-12 \mathrm{GHz}$ for the first time. The results obtained show stability in the values of the measured parameters. The melt blend $\mathrm{ZnO}$-PCL nanocomposites show great applicability in microwave attenuation. The substrates were fabricated at low temperature with easy preparation steps unlike other techniques. It was found that frequency is proportional to the magnetization, despite the use of semiconducting nanocomposites material. These relationships correspond to Snoek's limitation rule. From these results, it was clarified that ZnO-PCL nanocomposites having magnetization larger that Snoek's limit are suitable for high-microwave devices absorbing applications. The knowledge of the material properties is necessary to understand the potential use of these materials in high frequency applications. These substrates will be useful for many microwave applications such as antennas, inductors, filters, and absorbers.

\section{Conflict of Interests}

The authors declare that there is no conflict of interests regarding the publication of this paper.

\section{Acknowledgment}

The authors wish to thank the Universiti Putra Malaysia (UPM) for its support and for the provision of enabling environment to carry out this work.

\section{References}

[1] L. Cumbal, J. Greenleaf, D. Leun, and A. K. SenGupta, "Polymer supported inorganic nanoparticles: characterization and environmental applications," Reactive and Functional Polymers, vol. 54, no. 1-3, pp. 167-180, 2003.

[2] Y. D. Zhang, S. H. Wang, D. T. Xiao, J. I. Budnick, and W. A. Hines, "Nanocomposite $\mathrm{Co} / \mathrm{SiO}_{2}$ soft magnetic materials," IEEE Transactions on Magnetics, vol. 37, no. 4, pp. 2275-2277, 2001.

[3] S. Sindhu, S. Jegadesan, A. Parthiban, and S. Valiyaveettil, "Synthesis and characterization of ferrite nanocomposite spheres from hydroxylated polymers," Journal of Magnetism and Magnetic Materials, vol. 296, no. 2, pp. 104-113, 2006.

[4] M. Matsumoto and Y. Miyata, "Polymer absorbers containing magnetic particles: effect of polymer permittivity on wave absorption in the quasi-microwave band," Journal of Applied Physics, vol. 91, no. 12, pp. 9635-9637, 2002.

[5] R. Dai, G. Wu, W. Li, Q. Zhou, X. Li, and H. Chen, "Gelatin/carboxymethylcellulose/dioctyl sulfosuccinate sodium microcapsule by complex coacervation and its application for electrophoretic display," Colloids and Surfaces A: Physicochemical and Engineering Aspects, vol. 362, no. 1-3, pp. 84-89, 2010.

[6] J. Wang, F. Guan, L. Cui, J. Pan, Q. Wang, and L. Zhu, "Achieving high electric energy storage in a polymer nanocomposite at low filling ratios using a highly polarizable phthalocyanine interphase," Journal of Polymer Science B: Polymer Physics, vol. 52, no. 24, pp. 1669-1680, 2014.

[7] T. Tanaka, G. C. Montanari, and R. Mülhaupt, "Polymer nanocomposites as dielectrics and electrical insulation-perspectives for processing technologies, material characterization and future applications," IEEE Transactions on Dielectrics and Electrical Insulation, vol. 11, no. 5, pp. 763-784, 2004.

[8] R. Kochetov, Thermal and electrical properties of nanocomposites, including material processing [Ph.D. thesis], Lappeenranta University of Technology, Lappeenranta, Finland, 2012.

[9] Y. Yang, H. Chen, B. Zhao, and X. Bao, "Size control of ZnO nanoparticles via thermal decomposition of zinc acetate coated on organic additives," Journal of Crystal Growth, vol. 263, no. 1-4, pp. 447-453, 2004.

[10] R. Wu and C. Xie, "Formation of tetrapod $\mathrm{ZnO}$ nanowhiskers and its optical properties," Materials Research Bulletin, vol. 39, no. 4-5, pp. 637-645, 2004.

[11] A. Sharma and M. N. Afsar, "Accurate permittivity and permeability measurement of composite broadband absorbers at microwave frequencies," in Proceedings of the IEEE International Instrumentation and Measurement Technology Conference (I2MTC '11), pp. 1-6, IEEE, May 2011.

[12] A. Sharma and M. N. Afsar, "Microwave complex permeability and permittivity measurements of commercially available nano-ferrites," IEEE Transactions on Magnetics, vol. 47, no. 2, pp. 308-312, 2011.

[13] D. M. Pozar, Microwave Engineering, John Wiley \& Sons, 3rd edition, 2009.

[14] M. A. A. Rahman, S. Mahmud, A. Karim Alias, and A. F. M. Nor, "Effect of nanorod zinc oxide on electrical and optical properties of starch-based polymer nanocomposites," Journal of Physical Science, vol. 24, no. 1, pp. 17-28, 2013.

[15] X. Liu, X. Yin, L. Kong et al., "Fabrication and electromagnetic interference shielding effectiveness of carbon nanotube reinforced carbon fiber/pyrolytic carbon composites," Carbon, vol. 68, pp. 501-510, 2014. 
[16] M. Przybyszewska and M. Zaborski, "The effect of zinc oxide nanoparticle morphology on activity in crosslinking of carboxylated nitrile elastomer," eXPRESS Polymer Letters, vol. 3, no. 9, pp. 542-552, 2009.

[17] B. W. Li, Y. Shen, Z. X. Yue, and C. W. Nan, "Influence of particle size on electromagnetic behavior and microwave absorption properties of Z-type Ba-ferrite/polymer composites," Journal of Magnetism and Magnetic Materials, vol. 313, no. 2, pp. 322-328, 2007.

[18] S. Seema and M. V. N. A. Prasad, "Dielectric spectroscopy of nanostructured polypyrrole-NiO composites," Journal of Polymers, vol. 2014, Article ID 950304, 5 pages, 2014.

[19] P. Mitra and S. Mondal, "Structural and morphological characterization of $\mathrm{ZnO}$ thin films synthesized by SILAR," Progress in Theoretical and Applied Physics, vol. 1, pp. 17-31, 2013.

[20] A. Pakdel and F. E. Ghodsi, "Influence of drying conditions on the optical and structural properties of sol-gel-derived $\mathrm{ZnO}$ nanocrystalline films," Pramana-Journal of Physics, vol. 76, no. 6, pp. 973-983, 2011.

[21] H. Peng, Y. Han, T. Liu, W. C. Tjiu, and C. He, "Morphology and thermal degradation behavior of highly exfoliated CoAl-layered double hydroxide/polycaprolactone nanocomposites prepared by simple solution intercalation," Thermochimica Acta, vol. 502, no. 1-2, pp. 1-7, 2010. 

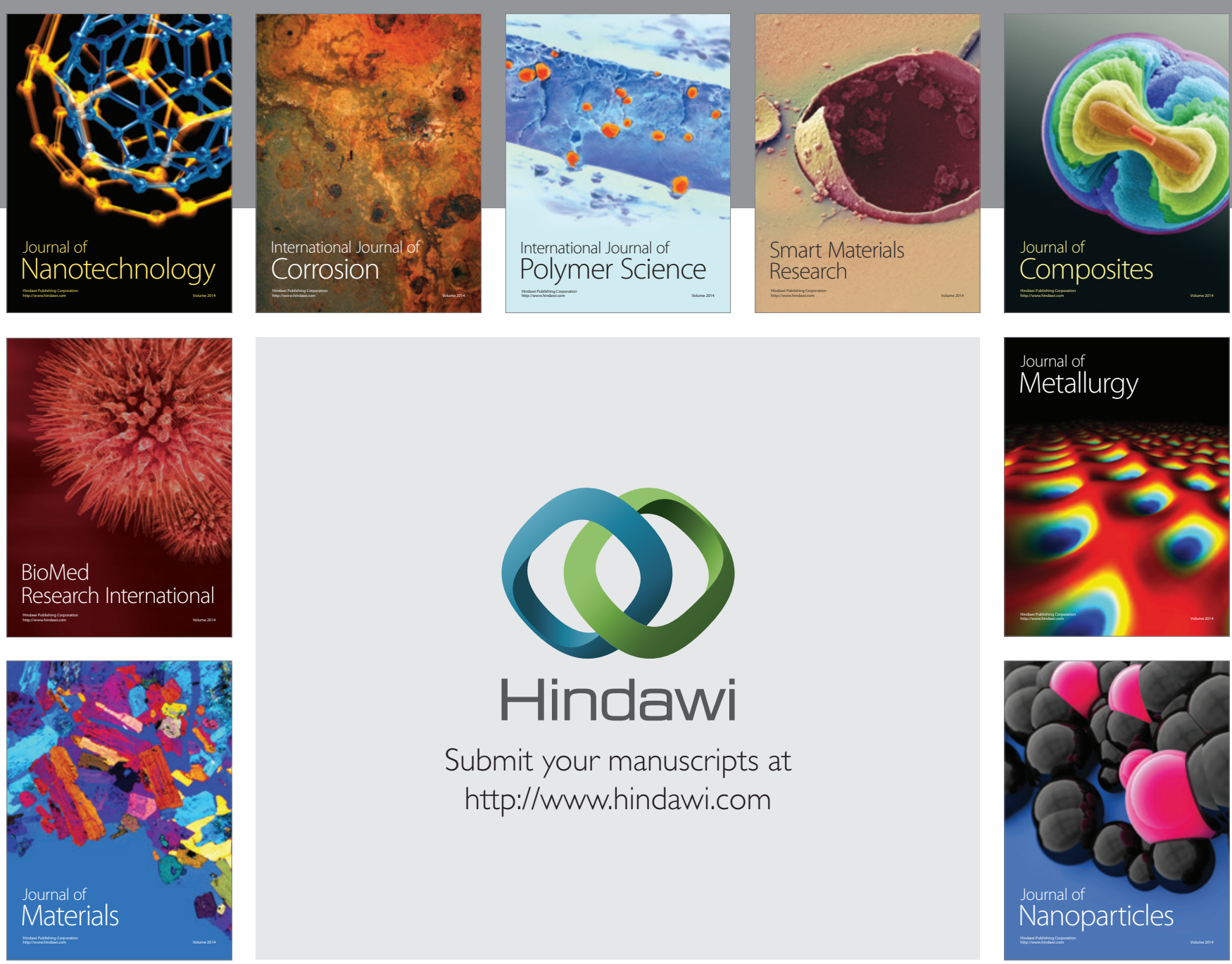

Submit your manuscripts at http://www.hindawi.com
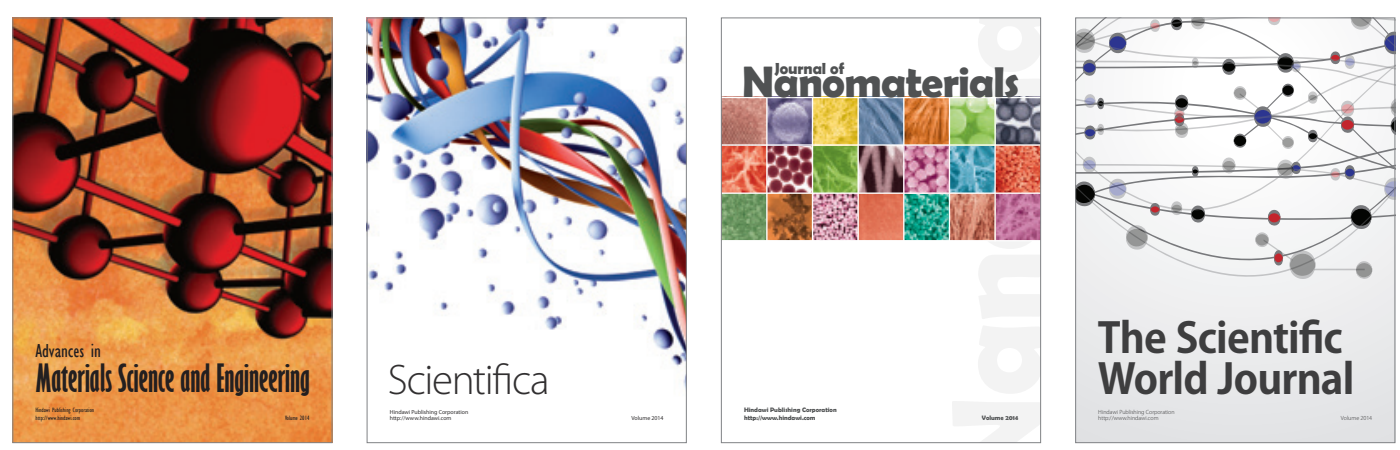

\section{The Scientific World Journal}
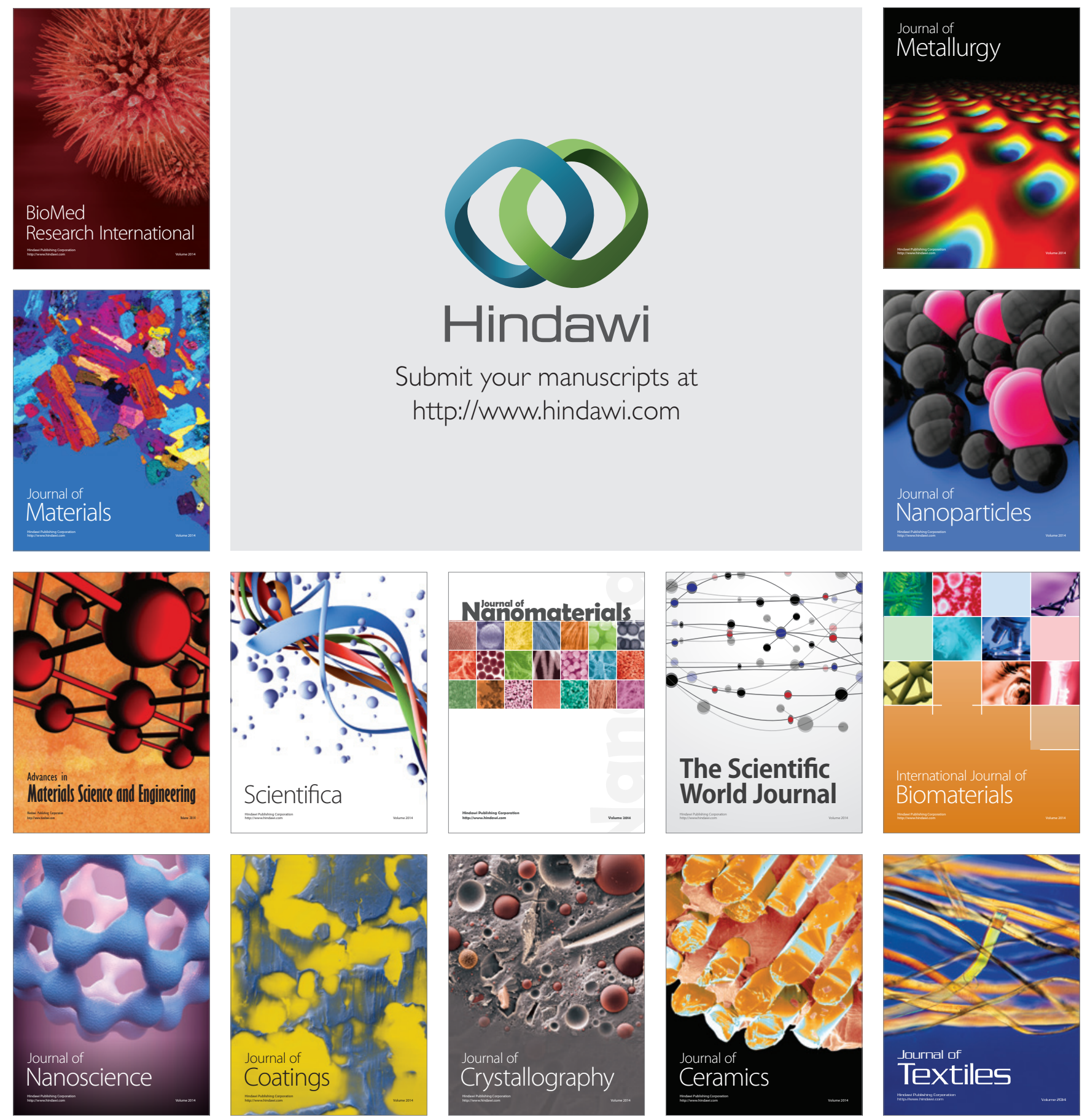